\title{
BMJ Open Mapping the use of soft systems methodology for change management in healthcare: a scoping review protocol
}

\author{
Hanna Augustsson, ${ }^{\circledR}$ Kate Churruca, Jeffrey Braithwaite
}

\begin{abstract}
To cite: Augustsson $\mathrm{H}$, Churruca K, Braithwaite J. Mapping the use of soft systems methodology for change management in healthcare: a scoping review protocol. BMJ Open 2019;9:e026028. doi:10.1136/ bmjopen-2018-026028

- Prepublication history for this paper is available online. To view these files, please visit the journal online (http://dx.doi. org/10.1136/bmjopen-2018026028).
\end{abstract}

Received 15 August 2018 Revised 5 March 2019 Accepted 8 March 2019

Check for updates

(C) Author(s) (or their employer(s)) 2019. Re-use permitted under CC BY-NC. No commercial re-use. See rights and permissions. Published by BMJ.

Australian Institute of Health Innovation, Macquarie University, Sydney, New South Wales, Australia

Correspondence to Dr Hanna Augustsson; hanna.augustsson@mq.edu.au

\begin{abstract}
Introduction It is notoriously challenging to implement evidence-based care and to update and improve healthcare practices. One reason for the difficulty is the complexity of healthcare and the powerful influence of context on implementation and improvement efforts. Thus, there is a need for multifaceted, flexible change methods that takes these complexities into consideration. One approach that has the potential in this regard is soft systems methodology (SSM). However, little is known about how SSM has been applied in healthcare settings, making it difficult to assess the usefulness of SSM for implementation science or improvement research. The aim of the proposed scoping review is to examine and map the use and outcomes of SSM in healthcare.

Methods and analysis The review will adapt the framework outlined by Arksey and 0'Malley (2005). Citations will be uncovered through a comprehensive database search of the peer-reviewed literature. Two reviewers will conduct a two-stage review and selection process where the titles/abstracts are examined followed by a screening of full texts of the selected citations. Reference lists of included citations will be snowballed to identify potential additional citations. Inclusion criteria are English language, peer-reviewed empirical papers focusing on the application of SSM in a healthcare setting. Both general information about the citations and information related to the objective of the review will be extracted from the included citations and entered into a data charting form. The extracted information will be reported in diagrams and tables and summarised to present a narrative account of the literature. The proposed review will provide information on the potential for using SSM to affect change in healthcare.

Ethics and dissemination No primary data will be collected, and thus ethical permission is unnecessary. Dissemination of results include peer-reviewed publications and conference presentations.
\end{abstract}

\section{INTRODUCTION}

Healthcare organisations are continuously required to implement new evidence and to improve their practices. However, improvement is exceedingly difficult. For instance, despite efforts to implement evidence-based care, only $55 \%-60 \%$ of patients receive recommended care in Australia ${ }^{12}$ and the USA. $^{3}$ These difficulties are related at least

\section{Strengths and limitations of this study}

The review will be limited to the peer-reviewed and English-speaking literature.

- It will not provide a definitive account of the effectiveness of soft systems methodology (SSM).

- The scoping methodology will allow information from a broad range of studies, using different designs and methods, to be included and synthesised.

- The review will provide a comprehensive overview of the application of SSM in healthcare and synthesise information that can inform assessment of the feasibility and usefulness of SSM in healthcare.

- The findings may highlight future directions for research on SSM in healthcare.

in part to the complexity of healthcare and the pervasive influence that context has on implementation and improvement efforts. Context includes the attitudes, perceptions and actions of the individuals involved, their collective cultural attributes and features of the inner and outer setting. ${ }^{4-6}$

Contextual factors manifest on multiple levels, are interwoven and interlinked and interact in unpredictable ways in influencing implementation. This makes it difficult to plan, execute and then predict how an intervention will be adopted and taken-up in any specific setting. ${ }^{78}$ Indeed, interventions that have been shown to be effective in one setting can fail to produce results in another setting. For instance, a meta-analysis comparing the effectiveness of internationally adopted interventions, culturally adapted interventions and novel interventions showed that novel and adapted interventions were more effective than adopted interventions. ${ }^{9}$ One explanation for this is that such interventions provide a better fit to the organisation's needs, culture, processes and structures and therefore are more likely to be embraced, implemented and sustained. ${ }^{51011}$

An important aspect of intervention fit is related to the individuals and groups that are involved in, and affected by, the intervention. 


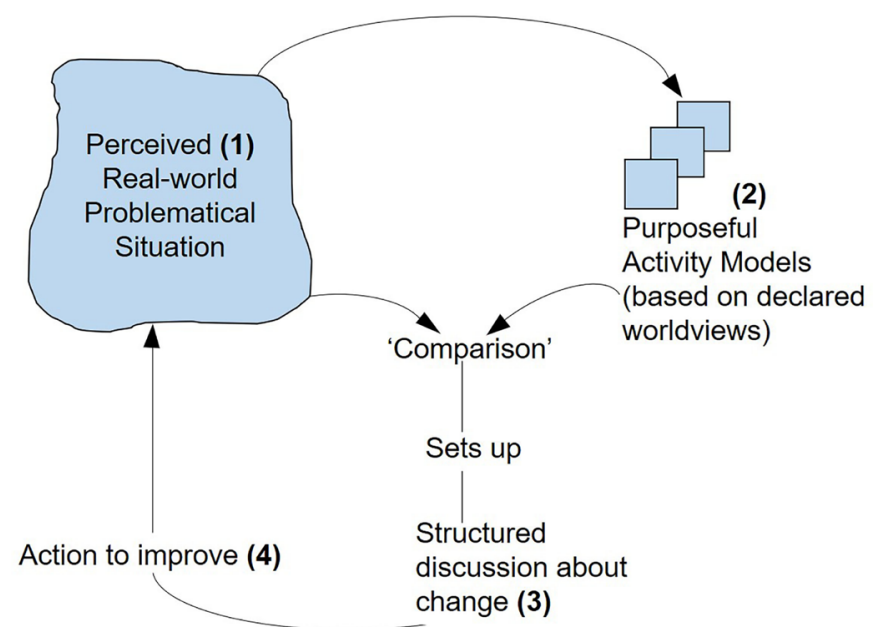

Figure 1 A generic SSM learning cycle. Source: Checkland and Poulter. ${ }^{19}$ Permission granted by John Wiley and Sons for use of this image. Licence number: 4403900665359.

They can ultimately contribute to the intervention's success or failure. ${ }^{4512}$ For the intervention to succeed, relevant individuals and stakeholder groups must recognise the problem and need for change, ${ }^{13}$ agree on solutions and put the intervention into practice. ${ }^{5} 12$ All-in-all, these issues underscore that improvement interventions cannot be separated from the context in which they are implemented. ${ }^{451415}$

This suggests that there is a need for more flexible, multifaceted and participatory change approaches that takes complexity into consideration rather than trying to simplify problems, interventions and contexts. ${ }^{6}{ }^{16-18}$ One approach that has been proposed to be useful in facilitating change in complex settings is soft systems methodology (SSM). ${ }^{19-21}$ SSM has several features that have been emphasised as important for responsive implementation and improvement interventions such as involving stakeholders in the change process, ${ }^{8}{ }^{17}$ factoring in the local context, ${ }^{4}{ }^{511}$ facilitating adaptation and ongoing learning ${ }^{41022}$ and taking a systems approach to change rather than trying to control all dependent variables or striving to affect change in one part of the system without recognising the interconnections with other parts. ${ }^{23} 24$

\section{SSM in brief}

SSM builds on systems theory. It is a methodology designed for tackling real-world problems that may be hard to define and where people may have divergent views on the problem in focus, and the objective of change, or both. SSM is described as a learning process that engages relevant stakeholders in a staged inquiry into a problematic situation with the aim of developing a purposeful model of activity that can be used for learning about the real-world and facilitating improvement of the problem. ${ }^{19-21}$ The learning cycle (figure 1) involves four activities: (1) Finding out about the problematic situation, including cultural and political dimensions. In this activity, the context of the problem situation and the interlinks between different contextual factors are explored. A rich picture is developed to illustrate this. (2) Formulating relevant purposeful activity models, that is, modelling how the activities in an improved situation could look. (3) Debating the situation, using the models to find changes that are desirable as well as contextually and culturally feasible, and seeking agreement between disparate views. (4) Taking action to bring about improvement by identifying opportunities for gain and progress based on the prior three activities, and testing changes as a basis for further learning. ${ }^{19}$

\section{SSM in healthcare}

A review conducted in 2007 showed that SSM had been used in a variety of areas, including healthcare. However, the majority of studies on SSM had been conducted in relation to development and implementation of information and communication technology, and environmental and ecological problem situations. ${ }^{25}$ A more recent review focused on the methodological aspects of the use of SSM in healthcare up to $2014 .{ }^{26}$ This review showed that SSM had been applied in various ways, including being modified and used in combination with other methods. However, there is, to the best of our knowledge, no recent review mapping the use of SSM in healthcare, especially in identifying the type of problems to which SSM has been applied, or the types of interventions and outcomes that have been reported following the use of SSM.

We propose a review to investigate these issues. Due to the likelihood of varying study designs and outcomes of studies describing the use of SSM, we consider a scoping review to be the optimal format. This will provide information about settings, the purpose of SSM use and an overview of the types of interventions that have been proposed and implemented as well as their reported outcomes. The findings will illustrate the extent to which SSM can be useful for the kinds of problematical situations healthcare is facing, and particularly it should unlock value in understanding contextually adapted change and improvement strategies.

\section{METHODS AND ANALYSIS}

We propose adapting for our purpose the framework of Arksey and $\mathrm{O}^{\prime}$ Malley ${ }^{27}$ for conducting scoping reviews. The framework includes five stages: (1) identifying the research question; (2) identifying relevant studies; (3) study selection; (4) charting the data; and (5) collating, summarising and reporting the results. The study protocol is outlined according to these five stages.

\section{Stage 1: identifying the research question Objectives}

The objective of the review is to examine and map the use and outcomes of SSM in the context of healthcare. The review will be guided by secondary questions: (1) In which countries and healthcare settings has SSM been applied? (2) How has SSM been applied, for example, for problem structuring, or for proposing or implementing 
interventions? (3) For what type of problems has SSM been used? (4) To what degree have stakeholders been involved and consulted in the SSM process? (5) What kinds of interventions have been proposed or implemented using SSM? (6) What kinds of outcomes have been reported following the use of SSM?

\section{Eligibility criteria}

Citations will be assessed against the following inclusion criteria: English-language, peer-reviewed, empirical research articles published in scholarly journals where the full text is available. The content of the citations should be on the application of SSM in a healthcare setting, including primary care, mental health, hospital care, residential age care, rehabilitation and community health facilities. Studies claiming to apply one or several elements from SSM will be included even if SSM has not been applied in its entirety. Studies using SSM, or parts of SSM, in combination with other methods will also be included. Citations focusing on the use of SSM in settings other than healthcare, for example, educational settings (including healthcare education) will be excluded. No date limit will be applied.

\section{Stage 2: identifying relevant studies}

The review will focus on peer-reviewed literature. The main identification strategy will be to search key electronic databases: Scopus, MEDLINE, Web of Science, CINAHL, EMBASE and PsycINFO. These databases were selected because they include a broad range of literature from different disciplines such as biomedicine, psychology, health services research and nursing.

The search strategy (table 1) will use the term 'soft systems method*' to identify citations referring to SSM. Search terms will be used to limit the search to the healthcare context, for example, health* and 'acute care'. The wildcard character, representing one or more other characters, allows variable endings of keywords, for example, healthcare, health system and healthcare organisation. In addition to the database search, the reference lists from the included citations will be snowball searched to identify additional citations. To reduce the likelihood that relevant articles are overlooked, we will also hand search reference lists of key methodological papers and review papers.

Because of the focus on a specific and named methodology, the search strategy can be defined well in advance,

\begin{tabular}{ll}
\hline Table 1 & Search strategy \\
\hline$\# 1$ & 'soft system method" (TITLE-ABS-KEY) \\
& AND \\
& $\begin{array}{l}\text { health* or hospital or 'acute care' or 'primary care' } \\
\text { or 'general practice' or 'aged care' or 'nurs* home' } \\
\text { or medic* or clinic* or nurs (TITLE-ABS-KEY) }\end{array}$ \\
& AND \\
$\# 3$ & LIMIT-TO (LANGUAGE, 'English') \\
\hline
\end{tabular}

enabling the identification of relevant citations and minimising citations not related to the scope of the review. The database searches will be made by one researcher (HA) and sample citations by another (KC) and include all citations published before the study cut-off date.

\section{Stage 3: study selection}

After duplicates have been removed in a structured process, ${ }^{28}$ all references will be imported into Rayyan, a web and mobile app, that organises and facilitates the initial screening of titles and abstracts. ${ }^{29}$ Two reviewers (HA and KC) will apply the inclusion and exclusion criteria to all the citations, both in the title and abstract review and in the full text review. To test the inclusion and exclusion criteria and ensure consensus on included citations, titles and abstracts from $10 \%$ of the identified references will be assessed by the two reviewers. Interrater agreement rates will be calculated using Cohen's kappa. ${ }^{30}$ Any discrepancies between authors concerning the inclusion or exclusion of citations will be resolved through discussion and, if necessary, a third researcher (JB) will be consulted. After this initial test, any adjustments or clarifications needed will be made to the inclusion and exclusion criteria. An agreement rate of 0.8 will be used as a target to ensure that the criteria are properly defined. The researchers will then review the remaining titles and abstracts. In the next step, the reviewers will assess the full texts of the included citations for final inclusion.

\section{Stage 4: charting the data}

An electronic data charting form will be developed to guide data charting from included citations. The form will be used to collect data relating to both general information about the citations such as publication year and authors as well as information related to the objective of the review (box 1). Charting the results in a scoping review can often be an iterative process since the review method may reveal additional data that may be relevant to extract. ${ }^{27}{ }^{31}$ The data charting form will be piloted by the two reviewers using a random selection of the citations and any changes needed will be made prior to data charting from the remaining citations. One of the reviewers (HA) will then independently chart the data from the remaining citations, with sample validation by $\mathrm{KC}$ and JB.

\section{Stage 5: collating, summarising and reporting the results}

A numerical overview of the extent, nature and distribution of the included studies will be summarised and reported in diagrams and tables to provide a synthesis of the literature on the use of SSM in healthcare. This may include in which countries and healthcare settings that SSM has been used and in what ways SSM has been used (eg, as a problem structuring method, for proposing/ implementing interventions or other applications). The extracted information will also be summarised to present a narrative account of the literature. Themes and categories will be both deductively developed, based on the 


\section{Box 1 Overview of data items for charting}

\section{Information to be charted:}

a. Author(s).

b. Publication year.

c. Title.

d. Aim.

e. Country of origin.

f. Type of healthcare setting(s).

g. Methods (design, data collection, participants)

h. Way of using soft systems methodology (SSM) (for problem structuring, for proposing/implementing interventions).

i. Type of problem that SSM has been applied to

j. Degree of stakeholder participation (eg, number of stakeholder groups that have been consulted in the different SSM activities).

k. Type of intervention (if applicable).

I. Type of outcomes reported (if applicable).

research questions, and inductively developed, based on the empirical data and determined during the analytical process. ${ }^{32}$ Examples include a narrative description of what type of problems SSM has been used to address, which interventions that have been proposed and/or implemented and reported outcomes.

\section{Patient and public involvement}

There was no patient or public involvement in the design of this scoping review protocol.

\section{CONCLUSION}

The complexity of the healthcare system and the challenge of implementing new evidence and improving care practices calls for multifaceted, flexible approaches to facilitate change that take these complexities into consideration. SSM is an approach that has the potential to facilitate change in complex settings and situations. However, how SSM has been used in healthcare, including the type of changes for which it may be useful, and outcomes reported following application of SSM, is not clear. As such, the proposed review aims to map the use of SSM and to explore the potential for using SSM to affect health systems change.

Contributors JB conceptualised the study. HA and JB developed the study design and methodology. HA drafted the initial manuscript, assisted by KC and JB. All authors had the opportunity to contribute to the final manuscript, edited it into its final form and approved the final submission.

Funding This work is supported by the National Health and Medical Research Council Partnership Centre grant in Health System Sustainability (ID:9100002).

Competing interests None declared.

Patient consent for publication Not required.

Ethics approval As no primary data will be collected, no ethical permission will be required. Dissemination of results include peer-reviewed publications and conference presentations.

Provenance and peer review Not commissioned; externally peer reviewed.

Open access This is an open access article distributed in accordance with the Creative Commons Attribution Non Commercial (CC BY-NC 4.0) license, which permits others to distribute, remix, adapt, build upon this work non-commercially, and license their derivative works on different terms, provided the original work is properly cited, appropriate credit is given, any changes made indicated, and the use is non-commercial. See: http://creativecommons.org/licenses/by-nc/4.0/.

\section{REFERENCES}

1. Braithwaite J, Hibbert PD, Jaffe A, et al. Quality of health care for children in Australia, 2012-2013. JAMA 2018;319:1113-24.

2. Runciman WB, Hunt TD, Hannaford NA, et al. CareTrack: assessing the appropriateness of health care delivery in Australia. Med J Aust 2012;197:100-5.

3. McGlynn EA, Asch SM, Adams J, et al. The quality of health care delivered to adults in the United States. N Engl J Med 2003;348:2635-45

4. Damschroder LJ, Aron DC, Keith RE, et al. Fostering implementation of health services research findings into practice: a consolidated framework for advancing implementation science. Implement Sci 2009;4:50.

5. Greenhalgh T, Robert G, Macfarlane F, et al. Diffusion of innovations in service organizations: systematic review and recommendations. Milbank Q 2004:82:581-629.

6. Grol R, Grimshaw J. From best evidence to best practice: effective implementation of change in patients' care. Lancet 2003;362:1225-30.

7. Nilsen P. Making sense of implementation theories, models and frameworks. Implement Sci 2015;10:53.

8. Durlak JA, DuPre EP. Implementation matters: a review of research on the influence of implementation on program outcomes and the factors affecting implementation. Am J Community Psychol 2008;41:327-50.

9. Sundell K, Beelmann A, Hasson $\mathrm{H}$, et al. Novel programs, international adoptions, or contextual adaptations? Meta-analytical results from German and Swedish intervention research. $J$ Clin Child Adolesc Psychol 2016:45:784-96.

10. Chambers DA, Glasgow RE, Stange KC. The dynamic sustainability framework: addressing the paradox of sustainment amid ongoing change. Implement Sci 2013;8:117.

11. Klein KJ, Sorra JS. The challenge of innovation implementation. Acad Manage Rev 1996;21:1055-80.

12. Weiner BJ. A theory of organizational readiness for change. Implement Sci 2009;4:67.

13. Kotter JP, Schlesinger LA. Choosing strategies for change. Harv Bus Rev 2008

14. Grimshaw JM, Eccles MP, Lavis JN, et al. Knowledge translation of research findings. Implement Sci 2012;7:50.

15. French SD, Green SE, O'Connor DA, et al. Developing theoryinformed behaviour change interventions to implement evidence into practice: a systematic approach using the Theoretical Domains Framework. Implement Sci 2012;7:38.

16. Braithwaite J, Churruca K, Long JC, et al. When complexity science meets implementation science: a theoretical and empirical analysis of systems change. BMC Med 2018;16:63.

17. von Thiele Schwarz U, Lundmark R, Hasson $H$. The Dynamic Integrated Evaluation Model (DIEM): achieving sustainability in organizational intervention through a participatory evaluation approach. Stress Health 2016;32:285-93.

18. Long KM, McDermott F, Meadows GN. Being pragmatic about healthcare complexity: our experiences applying complexity theory and pragmatism to health services research. BMC Med 2018;16:94.

19. Checkland P, Poulter J. Learning for action: a short definitive accoun of soft systems methodology and its use, for practitioners, teachers and students. Chichester: John Wiley and Sons Ltd, 2006.

20. Checkland P. Systems thinking, systems practice. Chichester: John Wiley and Sons, 1981.

21. Checkland P. Soft systems methodology: a thirty year retrospective. Syst Res Behav Sci 2000;17:S11-58.

22. Castro FG, Barrera M, Martinez CR. The cultural adaptation of prevention interventions: resolving tensions between fidelity and fit Prev Sci 2004:5:41-5.

23. Greenhalgh T. How to implement evidence-based healthcare. Wiley Blackwell: Oxford, 2018.

24. Plsek PE, Greenhalgh T. Complexity science: the challenge of complexity in health care. BMJ 2001;323:625-8.

25. van de Water H, Schinkel M, Rozier R. Fields of application of SSM: a categorization of publications. J Oper Res Soc 2007:58:271-87.

26. Powell J, Mustafee N. Widening requirements capture with soft methods: an investigation of hybrid M\&S studies in health care. $J$ Oper Res Soc 2017;68:1211-22. 
27. Arksey H, O'Malley L. Scoping studies: towards a methodological framework. Int J Soc Res Methodol 2005;8:19-32.

28. Karolinska Institutet University Library. De-duplication in Endnote. May 2018 https://kib.ki.se/en/search-evaluate/systematicreviews/deduplication-endnote.

29. Ouzzani M, Hammady H, Fedorowicz Z, et al. Rayyan-a web and mobile app for systematic reviews. Syst Rev 2016;5:210.
30. McHugh ML. Interrater reliability: the kappa statistic. Biochem Med 2012;22:276-82.

31. Peters M, Godfrey C, Mclnerney P, et al. The joanna briggs institute reviewers' manual 2015: methodology for JBI scoping reviews. Adelaide, Australia: The Joanna Briggs Institute, 2015.

32. Hsieh HF, Shannon SE. Three approaches to qualitative content analysis. Qual Health Res 2005;15:1277-88. 